\title{
The association between mechanical ventilator availability and mortality risk in intensive care patients with COVID-19: A national retrospective cohort study
}

Harrison Wilde (BSc) ${ }^{1 *}$, Thomas Mellan $(\mathrm{PhD})^{2^{*}}$, Iwona Hawryluk $(\mathrm{MSc})^{2}$, John M. Dennis $(\mathrm{PhD})^{3}$, Spiros Denaxas $(\mathrm{PhD})^{4,5,6}$, Christina Pagel $(\mathrm{PhD})^{7}$, Andrew Duncan $(\mathrm{PhD})^{4,8}$, Samir Bhatt $(\mathrm{PhD})^{2}$, Seth Flaxman $(\mathrm{PhD})^{8 \wedge}$, Bilal A Mateen $(\mathrm{MBBS})^{4,5,9^{\wedge * \dagger},}$, Sebastian J Vollmer $(\mathrm{PhD})^{1,4^{\wedge}}$

* These authors contributed equally to the manuscript

$\wedge$ Joint Senior Authors

1 University of Warwick, Department of Statistics. Address: Department of Statistics, University of Warwick, Coventry, CV4 7AL, UK

2 MRC Centre for Global Infectious Disease Analysis, Abdul Latif Jameel Institute for Disease and Emergency Analytics (J-IDEA), Imperial College, London, UK

3 University of Exeter Medical School. Address: Institute of Biomedical \& Clinical Science, RILD

Building, Royal Devon \& Exeter Hospital, Barrack Road, Exeter EX2 5DW, UK

4 The Alan Turing Institute. Address: British Library, 96 Euston Road, London, NW1 2DB, UK

5 University College London, Institute of Health Informatics. Address: University College London, 222

Euston Rd, London NW1 2DA, London, UK

6 Health Data Research UK. Address: Gibbs Building, 215 Euston Road, London, NW1 2BE, UK

7 University College London, Clinical Operational Research Unit. Address: University College London,

222 Euston Rd, London NW1 2DA, London, UK

8 Department of Mathematics, Imperial College, London, UK

9 The Wellcome Trust. Address: Gibbs Building, 215 Euston Road, London, NW1 2BE, UK

\section{Author for Correspondence (†)}

Dr. Bilal A. Mateen

Institute of Health Informatics, University College London

222 Euston Rd, London NW1 2DA, UK

Email: bilal.mateen@nhs.net

Tel: +44 (0)24 76574880

\section{Word Count: 2,006}

Kevwords: Critical Care, Hospital Mortality, Public Health Surveillance, Quality of Healthcare,

Coronavirus Infection 


\section{$\underline{\text { Summary Box }}$}

\section{What is already known on this topic:}

Pre-pandemic, higher occupancy of intensive care units was shown to be associated with increased mortality risk. However, there is limited data on the extent to which occupancy levels impacted patient outcomes during the COVID-19 pandemic, especially in light of the mobilisation of significant additional resources. A recent study from Belgium reported a $42 \%$ higher mortality during periods of ICU surge capacity deployment, although in the analysis surge capacity was evaluated only as a binary variable, and notably this contradicts earlier results from smaller studies in Australia and Wales, where no association between ICU occupancy and mortality was identified.

\section{What this study adds:}

The results of this study suggest that survival rates for patients with COVID-19 in intensive care settings appears to deteriorate as the occupancy of (surge capacity) beds compatible with mechanical ventilation (a proxy for operational pressure), increases. Moreover, this risk doesn't occur above a specific threshold, but rather appears linear; whereby going from $0 \%$ occupancy to $100 \%$ occupancy increases risk of mortality by $69 \%$ (after adjusting for relevant individual-level factors). Furthermore, risk of mortality based on occupancy on the date of recorded outcome is even higher; OR 2.98 (95\% posterior credible interval: $2.33-3.83)$. As such, this national-level cohort study of England provides compelling evidence for a relationship between occupancy and critical care mortality, and highlights the needs for decisive action to control the incidence and prevalence of COVID-19. 


\begin{abstract}
$\underline{\text { Abstract }}$
Objectives: To determine if there is an association between survival rates in intensive care units (ICU) and occupancy of the unit on the day of admission.

Design: National retrospective observational cohort study during the COVID-19 pandemic.

Setting: 90 English hospital trusts (i.e. groups of hospitals functioning as single operational units).

Participants: 6,686 adults admitted to an ICU in England between $2^{\text {nd }}$ April and $1^{\text {st }}$ December, 2020 (inclusive), with presumed or confirmed COVID-19, for whom data was submitted to the national surveillance programme and met study inclusion criteria.
\end{abstract}

\title{
Interventions: N/A
}

Main Outcomes and Measures: A Bayesian hierarchical approach was used to model the association between hospital trust level (mechanical ventilation compatible) bed occupancy, and in-hospital all-cause mortality. Results were adjusted for unit characteristics (pre-pandemic size), individual patient-level demographic characteristics (age, sex, ethnicity, time-to-ICU admission), and recorded chronic comorbidities (obesity, diabetes, respiratory disease, liver disease, heart disease, hypertension, immunosuppression, neurological disease, renal disease).

Results: 121,151 patient-days were observed, with a mortality rate of 20.8 per 1,000 patient days. Adjusting for patient-level factors, mortality was higher for admissions during periods of high occupancy (>85\% occupancy versus the baseline of 45 to $85 \%$ ) [OR 1.18 (95\% posterior credible interval (PCI): 1.00 to 1.38)]. In contrast, mortality was decreased for admissions during periods of low occupancy ( $<45 \%$ relative to the baseline) [OR 0.79 (95\% PCI: 0.69 to 0.90$)$ ].

Conclusion and Relevance: Increasing occupancy of beds compatible with mechanical ventilation, a proxy for operational strain, is associated with a higher mortality risk for individuals admitted to ICU. Public health interventions (such as expeditious vaccination programmes and non-pharmaceutical interventions) to control both incidence and prevalence of COVID-19, and therefore keep ICU occupancy low in the context of the pandemic, are necessary to mitigate the impact of this type of resource saturation.

\section{Trial Registration: N/A}




\section{$\underline{\text { Introduction }}$}

From the first reports of a novel coronavirus (SARS-CoV-2) in late 2019, to date, global mortality associated with the resultant disease (COVID-19) has exceeded 1.7 million people.[1] The virulence of the pathogen has prompted persistent concern about the ability of health services around the world to effectively care for the vast numbers of people affected.[2] These concerns are most relevant in the context of scarce resources (e.g., mechanical ventilation) required by patients in need of high-acuity support, which is relatively common in patients with COVID-19.[3] Notably, even with the introduction of non-pharmacological interventions such as stay-at-home orders, almost a third of all hospitals in England reached 100\% occupancy of their "surge" mechanical ventilation capacity (i.e., including the additional beds that were created through the re-allocation of resources) during the first wave of the pandemic.[4] England is now experiencing a second wave that is already worse than the first, with $40 \%$ more people in hospital, many hospitals overwhelmed and exhausted staff.[5] What remains unclear is whether and to what extent operating at these extremes of critical care occupancy impacted patient outcomes.

Pre-pandemic, higher occupancy levels in intensive care (which may reflect operational strain), was shown to be associated with higher mortality risk.[6] However, there is limited data on the extent to which occupancy levels impacted patient outcomes during the first wave of COVID-19.[7] A recent study from Belgium reported 42\% higher mortality during periods of ICU surge capacity deployment, although in the analysis surge capacity was evaluated only as a binary variable.[8] This contradicts earlier results from smaller studies in Australia and Wales, where no association between ICU occupancy and mortality was identified.[9,10] A better understanding of how operating under such extreme circumstances affects outcomes is crucial for two reasons: firstly, to allow hospitals to adapt practice to improve outcomes and secondly, to provide policy makers with more accurate information about the potential consequences of allowing health services to be overwhelmed. As such, in this study, we sought to evaluate the extent to which mortality risk in intensive care units (ICUs) over the course of the first wave of the COVID-19 pandemic in England could be explained by differences in occupancy. 


\section{$\underline{\text { Methods }}$}

\section{Data Sources}

Data on all intensive care unit (ICU) admissions across England were extracted from the COVID-19 Hospitalisation in England Surveillance System (CHESS).[11] Information on occupancy rates were extracted from the daily situation reports (i.e., 'SitRep'). ${ }^{4}$ Both datasets are mandatory regulatory submissions for all National Health Service (NHS) acute care providers in England, and further details about them can be found in the eMethods.

\section{Study population}

All ICU admissions reported to CHESS between $2^{\text {nd }}$ April $-1^{\text {st }}$ December (see eMethods for details on date selection), with presumed/confirmed COVID-19 (100\% tested positive during their admission; see eMethods for details on diagnosis), aged 18 - 99, non-pregnant, and with valid admission and occupancy data, were eligible for inclusion (eFigure 1).

\section{Patient and Public Involvement}

No patients were involved in the design, interpretation of the results, or dissemination of this study. However, we plan to disseminate the results to patients and the public in collaboration with several media partners whom have already agreed to report on this results.

\section{Recorded clinical features}

Patient-Level Data

Information extracted from CHESS about each patient comprised: administrative features (admitting trust, admission date), demographic characteristics (age, sex, ethnicity), recorded comorbidities (obesity, diabetes, asthma, other chronic respiratory disease, chronic heart disease, hypertension, immunosuppression due to disease or treatment, chronic neurological disease, chronic renal disease, chronic liver disease). Ethnicity was coded as white, Asian (Subcontinent and other), black, mixed, and other; comorbidities were recorded as binary indicator variables, with missing entries assumed to reflect the absence of a comorbidity. The appropriateness of this assumption in CHESS has been previously explored.[12]

\section{Occupancy Data}

Trusts are groups of geographically co-located hospitals that function as a single organisational unit within the UK's national healthcare system. Information extracted from SitRep about each trust comprised: pre-pandemic (January - March 2020) number of beds compatible with mechanical ventilation, the proportion of beds compatible with mechanical ventilation occupied on each day of the study period, and each trust's geographical region.[4] Linkage was carried out based on the trust that an individual was admitted to and the date of ICU admission in CHESS; patients in CHESS were matched via their admission date to the relevant occupancy information from the corresponding date in SitRep. 


\section{Outcome}

The primary outcome was in-hospital all-cause mortality; patients were followed up until death, discharge, transfer, or the final follow-up date of $22^{\text {nd }}$ December. Discharge and transfer were both treated as suggesting that the patient survived.

\section{Statistical Analysis}

Descriptive summaries were generated as median and interquartile ranges for continuous variables, and frequency and percentage incidence for categorical factors. Exploratory analyses included: the relationship of the COVID-19 epidemic curve to bed occupancy at a national level (eFigure 2); the distribution of missingness amongst patients and trusts (eFigure 3); variation in age and comorbidity burden over the first wave (eFigure 4); the impact of modelling continuous variables either linearly, through the use of threshold functions, or via (standard cubic) splines/smooths (eMethods).

A Bayesian hierarchical approach was used to model the association between the trust, group and patient-level factors and mortality risk. Specifically, a generalised additive mixed model was utilised, with intercept and slope coefficients for population and group level effects, and a Bernoulli likelihood with logit function to link to mortality outcome. Coefficients were inferred by Markov chain Monte Carlo sampling, using Stan (CmdStan V2.25.0), with a model specified using BRMS (V2.14.4) in R (V4.0.3).[13-15] Further information on the Bayesian prior specification and modelling methods are reported in the eMethods.

As secondary analyses, two potential interactions were assessed: 1) baseline trust size and occupancy; 2) patient age and occupancy (results not reported due to insignificance). We also assessed the association of occupancy on the recorded outcome date with mortality, and occupancy expressed in terms of pre-pandemic ICU size. Several sensitivity analyses were carried out: 1) filtering for different degrees of missingness of patient-level comorbidity data at trust-level; 2) including all individuals still on the unit as of $22^{\text {nd }}$ December (and assuming they survive; $\mathrm{n}=495) ; 3$ ) including all individuals with a known outcome but no date whom were excluded in the data cleaning process $(n=8)$; 4) a random effect for all patient post $16^{\text {th }}$ June 2020 (i.e. the date of the Recovery Trials press-release regarding the effectiveness of dexamethasone); 5) adjusting for week of admission; 6) adjusting for trust and region as random effects; 7) additional patient-level factors: time from hospital admission to ICU admission, chronic liver disease and obesity; see eMethods for justifications. 


\section{$\underline{\text { Results }}$}

6,686 individuals were included in this study following application of the inclusion/exclusion criteria (see eFigure 1), of whom 2,517 (37.6\%) died. In total, 121,151 (median 12 days per patient; IQR: 6 - 23) patientdays were observed, equating to a mortality rate of 20.8 per 1,000 patient days. A full summary of the recorded patient-level characteristics is reported in Table 1.

\section{Mechanical ventilator occupancy rate on the day of admission is associated with mortality risk}

For high occupancy rates $(85-100 \%)$, the unadjusted odds ratio (OR) for mortality based on the mechanical ventilator occupancy rate on the day of admission was 1.14 (95\% posterior credibility interval (PCI): 1.00 1.33), relative to the baseline of $45-85 \%$. For low occupancy rates $(0-45 \%)$, the unadjusted odds ratio was 0.79 (95\% PCI: $0.70-0.90)$, relative to the baseline of $45-85 \%$. Following adjustment for patient-level factors (age, sex, ethnicity, and comorbidities), the OR was 1.18 (95\% PCI: $1.00-1.38$ ) for high occupancy rates, and 0.79 (95\% PCI: $0.69-0.90$ ) for low occupancy rates. Figure 1 illustrates the posterior probabilities for the fully adjusted ORs (see eFigure $5 \& 6$ for other model coefficients). To aid interpretation, the difference in risk for a 70 -year-old man with no comorbidities being admitted during a period of high versus low occupancy is equivalent to the risk of them being over a decade older (Figure 2). Sensitivity analyses as detailed in eTable 1 were all concordant with the primary analysis.

\section{Mortality risk increases linearly with admission and date-of-outcome specific occupancy}

The fully adjusted OR for mortality (Figure 3), using occupancy on the day of admission coded as a continuous linear variable ranging from 0 to 1 was 1.69 (95\% PCI: $1.32-2.18)$. Moreover, using the bed occupancy from each individuals' outcome date identified an even larger association (full model specification in eTable 2), OR 2.98 (95\% PCI: $2.33-3.83)$.

\section{Larger ICUs experience exaggerated impacts of extremes of mechanical ventilator occupancy rates}

Although pre-pandemic number of beds did not substantially alter the OR of occupancy as a sensitivity analysis (eTable 1), it did appear independently informative. An increase in pre-pandemic ICU size by 25 beds compatible with mechanical ventilation was associated with a $22 \%$ decrease in risk of mortality (eTable 3 ). The introduction of an interaction term between pre-pandemic size and occupancy identified that larger ICUs experience exaggerated impacts of extremes of mechanical ventilator occupancy rates (eFigure 8). 
medRxiv preprint doi: https://doi.org/10.1101/2021.01.11.21249461; this version posted January 19, 2021. The copyright holder for this preprint (which was not certified by peer review) is the author/funder, who has granted medRxiv a license to display the preprint in perpetuity.

It is made available under a CC-BY 4.0 International license.

Table 1: Characteristics of the study cohort stratified by occupancy on the day of admission.

\begin{tabular}{rrrr} 
& & Occupancy & \\
\hline $\mathbf{0 - 4 5 \%}$ & $\mathbf{4 5 - \mathbf { 8 5 } \%}$ & $\mathbf{8 5 - \mathbf { 1 0 0 \% }}$ & Overall \\
$(\mathrm{n}=1,563)$ & $(\mathrm{n}=4,096)$ & $(\mathrm{n}=1,027)$ & $(\mathrm{n}=6,686)$
\end{tabular}

Age in Years

$\begin{array}{lllll}\text { Median [IQR] } & 60[51,69] & 60[51,68] & 60[52,67] & 60[51,68]\end{array}$

Time in Days to ICU from Hospital Admission

$\begin{array}{lllll}\text { Median [IQR] } & 1[0,3] & 1[0,3] & 1[0,3] & 1[0,3]\end{array}$

Age Group

$\begin{array}{lrrrr}18-24 & 11(0.7) & 41(1.0) & 12(1.2) & 64(1.0) \\ 25-34 & 68(4.4) & 138(3.4) & 35(3.4) & 241(3.6) \\ 35-44 & 134(8.6) & 349(8.5) & 81(7.9) & 564(8.4) \\ 45-54 & 324(20.7) & 828(20.2) & 220(21.4) & 1,372(20.5) \\ 55-64 & 438(28.0) & 1,283(31.3) & 328(31.9) & 2,049(30.6) \\ 65-74 & 381(24.4) & 984(24.0) & 265(25.8) & 1,630(24.4) \\ 75-84 & 185(11.8) & 407(9.9) & 76(7.4) & 668(10.0) \\ 85+ & 22(1.4) & 66(1.6) & 10(1.0) & 98(1.5)\end{array}$

Sex

$\begin{array}{lrrrr}\text { Female } & 537(34.4) & 1,269(31.0) & 304(29.6) & 2,110(31.6) \\ \text { Male } & 1,026(65.6) & 2,827(69.0) & 723(70.4) & 4,576(68.4)\end{array}$

Ethnicity

$\begin{array}{lrrrr}\text { White } & 1,068(68.3) & 2,369(57.8) & 460(44.8) & 3,897(58.3) \\ \text { Asian Subcontinent } & 115(7.4) & 436(10.6) & 127(12.4) & 678(10.1) \\ \text { Asian (Other) } & 75(4.8) & 259(6.3) & 84(8.2) & 418(6.3) \\ \text { Black } & 60(3.8) & 262(6.4) & 110(10.7) & 432(6.5) \\ \text { Mixed } & 12(0.8) & 73(1.8) & 37(3.6) & 122(1.8) \\ \text { Other } & 64(4.1) & 244(6.0) & 67(6.5) & 375(5.6) \\ \text { Missing } & 169(10.8) & 453(11.1) & 142(13.8) & 764(11.4)\end{array}$

Obesity

$\begin{array}{lrrrr}\text { Obese } & 646(41.3) & 1,576(38.5) & 433(42.2) & 2,655(39.7) \\ \text { Non-Obese } & 520(33.3) & 1,565(38.2) & 272(26.5) & 2,357(35.3) \\ \text { Missing } & 397(25.4) & 955(23.3) & 322(31.4) & 1,674(25.0)\end{array}$

Comorbidity

$\begin{array}{lrrrr}\text { Diabetes } & 358(22.9) & 1,087(26.5) & 282(27.5) & 1,727(25.8) \\ \text { Chronic Respiratory Disease(s) } & 381(24.4) & 847(20.7) & 208(20.3) & 1,436(21.5) \\ \text { Chronic Heart Disease } & 194(12.4) & 499(12.2) & 98(9.5) & 791(11.8) \\ \text { Chronic Renal Disease } & 123(7.9) & 346(8.4) & 70(6.8) & 539(8.1) \\ \text { Chronic Neurological Disease } & 103(6.6) & 198(4.8) & 35(3.4) & 336(5.0) \\ \text { Chronic Liver Disease } & 83(5.3) & 84(2.1) & 16(1.6) & 183(2.7) \\ \text { Immunosuppressive Disease } & 62(4.0) & 143(3.5) & 20(1.9) & 225(3.4) \\ \text { Hypertension } & 524(33.5) & 1,446(35.3) & 305(29.7) & 2,275(34.0) \\ & & & & \\ \text { Crude rate/Unadjusted } & 513(32.8) & 1,571(38.4) & 433(42.2) & 2,517(37.6)\end{array}$

Legend: Continuous covariates are presented with their median and interquartile range, whilst categorical covariates are presented with their frequency and within column percentage prevalence. 


\section{$\underline{\text { Discussion }}$}

The results of this study highlight a potential major impact of operational pressure on patient survival during the COVID-19 pandemic. Survival rates for patients with COVID-19 in intensive care settings appears to deteriorate as the occupancy of beds compatible with mechanical ventilation (a proxy for operational pressure),[17] increases. These observations are consistent with the aforementioned Belgian study,[8] except that our results suggest a linear association rather than single step increase at a specific threshold. Moreover, our findings also corroborate previous reports of an association between larger ICUs and lower COVID-19 mortality.[18] However, we additionally observe an interaction with (pre-pandemic) unit size, whereby larger ICUs experience more exaggerated impacts from both higher and lower (surge) occupancy rates. Although, It is unclear from our data what is driving this heterogeneity. Finally, these results might partially explain the decreased mortality rate seen in the latter half of the first wave in the UK,[19] where occupancy rates were much lower than at the peak.[4] Importantly, the average number of co-morbidities per patient appears to rise when occupancy decreases (eFigure 4), suggesting if there was implicit/soft triaging it would not have been in a manner that would explain the observed effect (i.e. more co-morbidities are generally thought to be associated with a worse outcome). However, more research is necessary to definitively exclude this potential explanation for the results.

\section{Strengths and Limitations}

The strengths of this study are the national cohort of patient-level data with extensive capture of admissions, [20] coupled with a rigorous modelling method (eTable $4 \&$ eMethods). Limitations include a lack of physiological data, limiting our ability to adjust for differences in severity upon admission. However, it is worth noting that previous studies using linked CHESS data from the first wave of the pandemic did not find between centre variation in severity scores (e.g. mean APACHE-II) to be associated with mortality risk.[21] Moreover, the characterisation of operational strain as a function of surge occupancy likely fails to fully reflect the complexity of using non-specialist staff and other resource allocation issues present when 'creating' new ICU beds (see eTable 5 using an alternative definition of occupancy based on baseline capacity; mortality risk given this linear continuous factor was 1.27 (95\% PCI: 1.13 - 1.43). Finally, we lack clear 30-day outcome data for discharged and transferred individuals, and thus were forced to model under a naïve assumption that these individuals survived, which may have impacted our estimates of the risk.

\section{$\underline{\text { Implications for Policy Makers and Clinicians }}$}

In summary, our study highlights the importance of public health interventions (such as expeditious vaccination programmes and non-pharmacological interventions), to control both incidence and prevalence of COVID-19, and therefore actively manage ICU occupancy, as there is evidence of direct harm to patients as a consequence of saturation. This is especially relevant given the identification of a new strain of COVID-19 with a potentially increased risk of transmission,[22] coupled with observations that second wave-related operational pressures (including bed occupancy rates) in England have exceeded levels seen during the first wave of the pandemic,[5] suggesting immediate and decisive action is necessary. 


\section{Acknowledgements}

HW is supported by the Feuer International Scholarship in Artificial Intelligence. TAM, IH and SB acknowledge funding from the MRC Centre for Global Infectious Disease Analysis (reference no. MR/R015600/1), jointly funded by the UK Medical Research Council (MRC) and the UK Foreign, Commonwealth \& Development Office (FCDO), under the MRC/FCDO Concordat agreement and is also part of the EDCTP2 programme supported by the European Union. SB and SF were support by the MRC to undertake research on COVID-19 (MR/V038109/1). SB acknowledges funding from The Academy of Medical Sciences (SBF004/1080), Bill \& Melinda Gates Foundation (CRR00280) and Imperial College Healthcare NHS Trust - BRC Funding (RDA02). JMD is supported by an Independent Fellowship funded by Research England's Expanding Excellence in England (E3) fund. SJV, and BAM are supported by The Alan Turing Institute (EPSRC grant EP/N510129/). SJV is also supported by the University of Warwick IAA funding. SF is supported by EPSRC (EP/V002910/1). AD is supported by Wave 1 of the UKRI Strategic Priorities Fund under the EPSRC Grant EP/T001569/1.

\section{Contributors}

BAM conceived the study, with input from SJV, and JMD. HW, JMD, and SJV prepared the data. HW, TM, and IH undertook the analysis, with input from SJV, SF, and BAM. All authors contributed to the development of the visualisations, and interpretation of results. BAM, HW, and TM drafted the manuscript. All authors critically revised the article and approved the final version for submission.

\section{Data Access Statement}

Data cannot be shared publicly as it was collected by Public Health England (PHE) as part of their statutory responsibilities, which allows them to process patient confidential data without explicit patient consent. Data utilised in this study were made available through an agreement between the University of Warwick and PHE. Individual requests for access to CHESS data are considered directly by PHE (contact via covid19surv@phe.gov.uk).

\section{Role of the funding source}

This study received no direct funding. The individual authors' funder had no role in the design of the study, the analysis, or the formulation of the manuscript.

\section{Declaration of interest}

SJV declares funding from IQVIA and Microsoft. BAM is an employee of the Wellcome Trust, and holds a Wellcome funded honorary post at University College London for the purposes of carrying out independent research; the views expressed in this manuscript do not necessarily reflect the views or position of the Wellcome Trust. All other authors declare no competing interests.

\section{Transparency Statement}

The senior authors (SJV, BAM) had full access to all data and had final responsibility for the decision to submit for publication. SJV and BAM affirm that the manuscript is an honest, accurate, and transparent account of the 
medRxiv preprint doi: https://doi.org/10.1101/2021.01.11.21249461; this version posted January 19, 2021. The copyright holder for this preprint (which was not certified by peer review) is the author/funder, who has granted medRxiv a license to display the preprint in perpetuity.

It is made available under a CC-BY 4.0 International license .

study being reported; that no important aspects of the study have been omitted; and that any discrepancies from the study as originally planned have been explained.

\section{Ethics Approval}

The study was reviewed and approved by the Warwick Biosciences Research Ethics Committee (BSREC 120/19-20-V1.1) and sponsorship is being provided by University of Warwick (SOC.28/19-20). 


\section{$\underline{\text { References }}$}

1. World Health Organisation. COVID-19 Weekly Epidemiological Update - 29 December 2020.; 2020. https://www.who.int/publications/m/item/weekly-epidemiological-update---29-december-2020. Accessed January 1, 2021.

2. Ferguson N, Laydon D, Nedjati Gilani G, et al. Report 9: Impact of non-pharmaceutical interventions (NPIs) to reduce COVID19 mortality and healthcare demand. https://www.imperial.ac.uk/media/imperial-college/medicine/mrc-gida/2020-03-16-COVID19-Report9.pdf Accessed January 1, 2021.

3. Docherty A, Harrison E, Green C et al. Features of $20 \square 133$ UK patients in hospital with covid-19 using the ISARIC WHO Clinical Characterisation Protocol: prospective observational cohort study. BMJ. 2020:m1985

4. Mateen BA, Wilde H, Dennis JM, et al. A geotemporal survey of hospital bed saturation across England during the first wave of the COVID-19 Pandemic. BMJ Open [In Press].

5. Mahase E. Covid-19: Hospitals in crisis as ambulances queue and staff are asked to cancel leave. BMJ 2020;371:m4980. doi: https://doi.org/10.1136/bmj.m4980

6. Gattinoni L, Radrizzani D, Simini B, et al. Volume of activity and occupancy rate in intensive care units. Association with mortality. Intensive care medicine. 2004 Feb 1;30(2):290-7.

7. Xie Y, Bowe B, Maddukuri G, Al-Aly Z. Comparative evaluation of clinical manifestations and risk of death in patients admitted to hospital with covid-19 and seasonal influenza: cohort study. bmj. 2020 Dec 15;371. doi: https://doi.org/10.1136/bmj.m4677

8. Taccone FS, Vangoethem N, Depauw R, et al. The role of organizational characteristics on the outcome of COVID-19 patients admitted to the ICU in Belgium. The Lancet Regional Health-Europe. $2020 \operatorname{Dec} 23: 100019$.

9. Burrell AJ, Pellegrini B, Salimi F, et al. Outcomes of COVID-19 patients admitted to Australian intensive care units during the early phase of the pandemic. Med J Aust. 2020 Dec 12;1. doi: https://doi.org/10.5694/mja2.50883

10. Baumer T, Phillips E, Dhadda A, Szakmany T. Epidemiology of the First Wave of COVID-19 ICU Admissions in South Wales-The Interplay Between Ethnicity and Deprivation. Front Med (Lausanne). 2020;7. doi:10.3389/fmed.2020.569714

11. Public_Health_England.COVID-19 Hospitalisation in England Surveillance System (CHESS) 2020. https://www.england.nhs.uk/coronavirus/wp-content/uploads/sites/52/2020/03/phe-letter-to-trusts-redaily-covid-19-hospital-surveillance-11-march-2020.pdf. Accessed January 1, 2021.

12. Dennis JM, Mateen BA, Sonabend R, et al. Type 2 Diabetes and COVID-19-Related Mortality in the Critical Care Setting: A National Cohort Study in England, March-July 2020. Diabetes Care. 2020 Oct 23;44(1):50-7.

13. Carpenter B, Gelman A, Hoffman MD, Lee D, Goodrich B, Betancourt M, Brubaker M, Guo J, Li P, Riddell A. Stan: A probabilistic programming language. Journal of statistical software. 2017 Jan $1 ; 76(1)$. 
14. Bürkner PC. brms: An R package for Bayesian multilevel models using Stan. Journal of statistical software. 2017 Aug 29;80(1):1-28.

15. Development Core Team RR. R: A language and environment for statistical computing. 2011.

16. Spiegelhalter D. How old are you, really? Communicating chronic risk through 'effective age' of your body and organs. BMC Medical Informatics and Decision Making. 2016;16(1):104.

17. Bagshaw SM, Wang X, Zygun DA, et al. Association between strained capacity and mortality among patients admitted to intensive care: a path-analysis modeling strategy. Journal of critical care. 2018 Feb $1 ; 43: 81-7$.

18. Gupta S, Hayek SS, Wang W, et al. Factors associated with death in critically ill patients with coronavirus disease 2019 in the US. JAMA internal medicine. 2020 Nov 1;180(11):1436-46.

19. Dennis JM, McGovern AP, Vollmer SJ, Mateen BA. Improving survival of critical care patients with coronavirus disease 2019 in England: a national cohort study, March to June 2020. Critical Care Medicine. 2020 Dec 8.

20. Doidge JC, Gould DW, Ferrando-Vivas P, et al. Trends in intensive care for patients with COVID-19 in England, Wales and Northern Ireland. American journal of respiratory and critical care medicine. $2020 \operatorname{Sep} 9(\mathrm{ja})$.

21. Qian Z, Alaa AM, van der Schaar M, Ercole A. Between-centre differences for COVID-19 ICU mortality from early data in England. Intensive Care Med. 2020.

22. Volz E, Mishra S, Chand M, et al. Report 42 - Transmission of SARS-CoV-2 Lineage B.1.1.7 in England: insights from linking epidemiological and genetic data. Medrxiv. $31^{\text {st }}$ Dec 2020. doi: https://www.medrxiv.org/content/10.1101/2020.12.30.20249034v2 


\section{Figure Legends}

Figure 1: The adjusted odds ratios for the risk of mortality based on different ICU bed occupancy rates (treated as a three-level categorical variable)

The full posterior distribution of the odds ratio (OR) for mortality given low occupancy 0 - 45\% (Top; Green), and high occupancy $85-100 \%$ (Bottom; Red) are presented. The PCIs presented are equally tailed credibility intervals for the posterior odds ratio distributions. The outer (less saturated) interval is the 95\% PCI, and the inner (more saturated) interval shows the 90\% PCI. The reference category is 45 - 85\% occupancy. Exact values are tabulated below.

\begin{tabular}{|c|c|c|c|c|c|}
\hline & & \multicolumn{3}{|c|}{ Posterior Credible Intervals } & \multirow[t]{2}{*}{ Median } \\
\hline & \multicolumn{2}{|r|}{$95 \%$} & \multicolumn{2}{|r|}{$90 \%$} & \\
\hline & Lower & Upper & Lower & Upper & \\
\hline \multicolumn{6}{|l|}{ Occupancy } \\
\hline $0-45 \%$ & 0.69 & 0.90 & 0.70 & 0.88 & 0.79 \\
\hline $85-100 \%$ & 1.00 & 1.38 & 1.02 & 1.35 & 1.18 \\
\hline
\end{tabular}

Figure 2: The increase in mortality risk associated with admission to intensive care during periods of different occupancy rates, expressed in terms of the equivalent increase in years of age.

The plot illustrates the number of years of additional age that ICU admission on a day with each different mechanical ventilation bed occupancy rate equates to. For example, an individual with a chronological age of 40, has an effective age of 31 in a low occupancy setting (Green), and 45 in a high occupancy setting (Orange). Both of the aforementioned comparisons are relative to the baseline occupancy of 45 - 85\%). A comparison of the difference in risk between being admitted to the highest occupancy range relative to the lowest is shown in (Red), and for a 40-year-old is equivalent to an increase in their age by 11 years. The method for generating this specific type of plot is described in detail in the eMethods and via eFigure 7.[16]

Figure 3: The adjusted odds ratios for the risk of mortality based on ICU bed occupancy (treated as a $\underline{\text { linear continuous variable) }}$ on the day of admission (top) and each individual's recorded outcome date $\underline{\text { (bottom) }}$

The full posterior distribution of the odds ratio (OR) for mortality given occupancy on the date of ICU admission (Top; Purple), and occupancy on the date of each individual's recorded outcome (Bottom; Blue) are presented. The PCIs presented are equally tailed credibility intervals for the posterior odds ratio distributions. Occupancy was specified without multiplying out by 100 (i.e., $20 \%=0.20$ ), therefore the odds ratio is for a step from $0 \%$ to $100 \%$ (i.e., 0.0 to 1.0 ). Exact values are tabulated below.

\begin{tabular}{|c|c|c|c|c|c|}
\hline & & \multicolumn{3}{|c|}{ Posterior Credible Intervals } & \multirow[t]{2}{*}{ Median } \\
\hline & \multicolumn{2}{|r|}{$95 \%$} & \multicolumn{2}{|r|}{$90 \%$} & \\
\hline & Lower & Upper & Lower & Upper & \\
\hline \multicolumn{6}{|l|}{ Occupancy } \\
\hline Continuous Linear (Admission Date) & 1.32 & 2.18 & 1.37 & 2.09 & 1.69 \\
\hline Continuous Linear (Outcome Date) & 2.33 & 3.83 & 2.42 & 3.68 & 2.98 \\
\hline
\end{tabular}




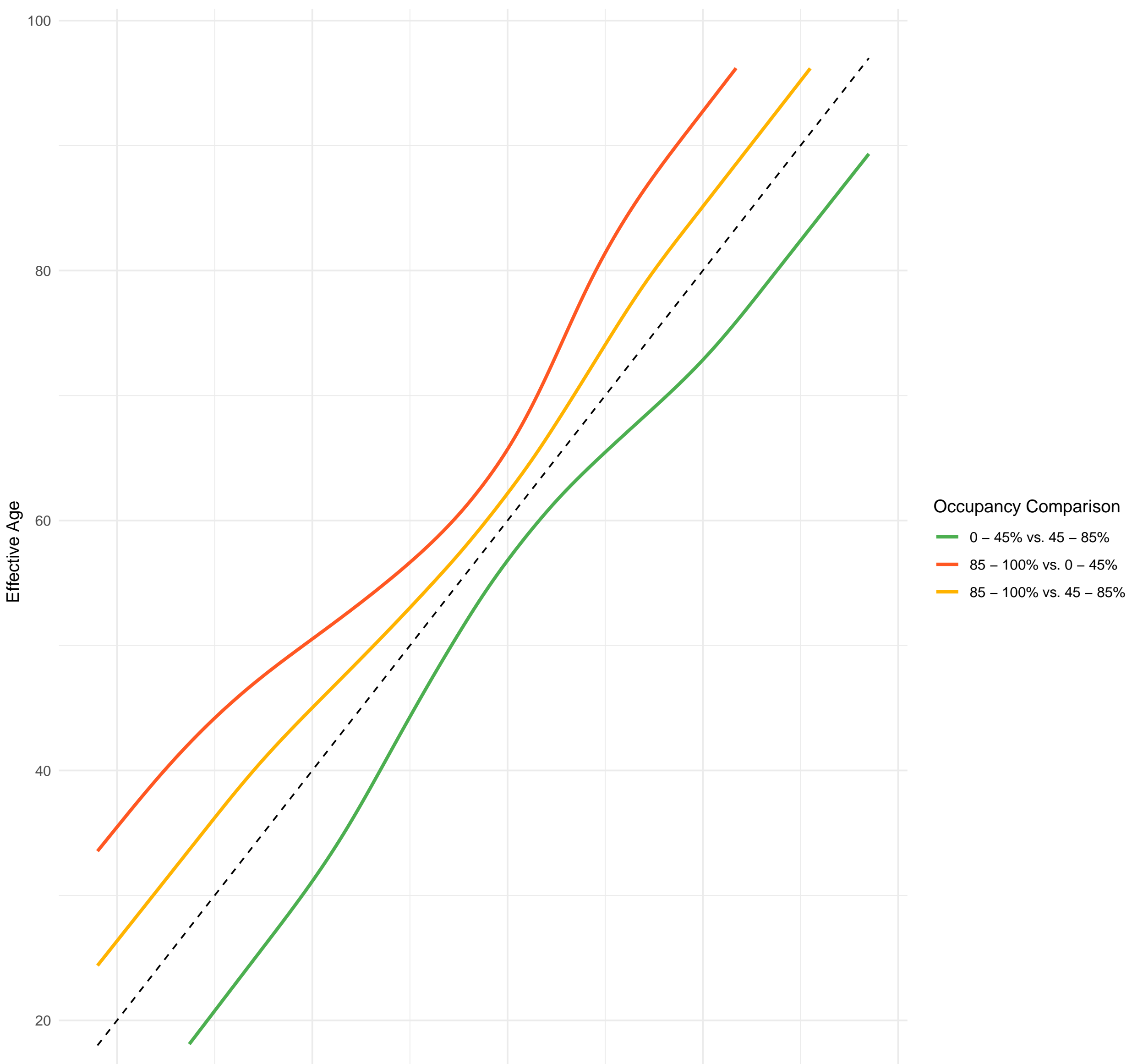




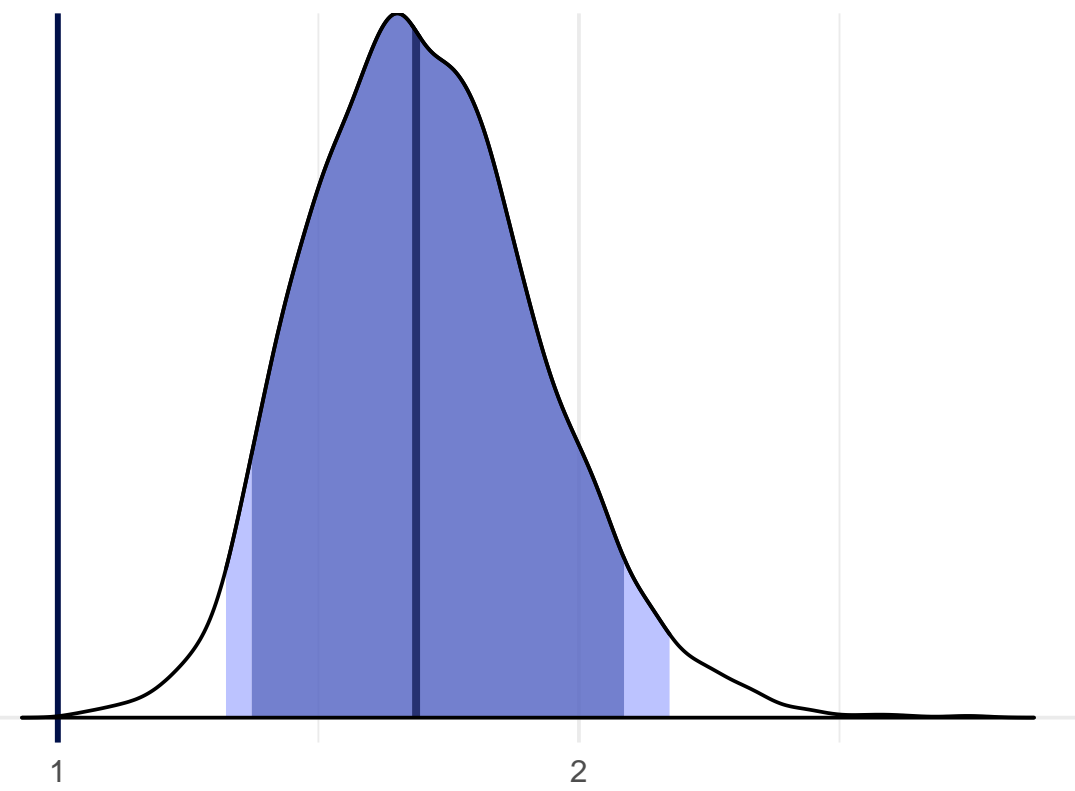

Occupancy on Each Individual's Recorded Outcome Date

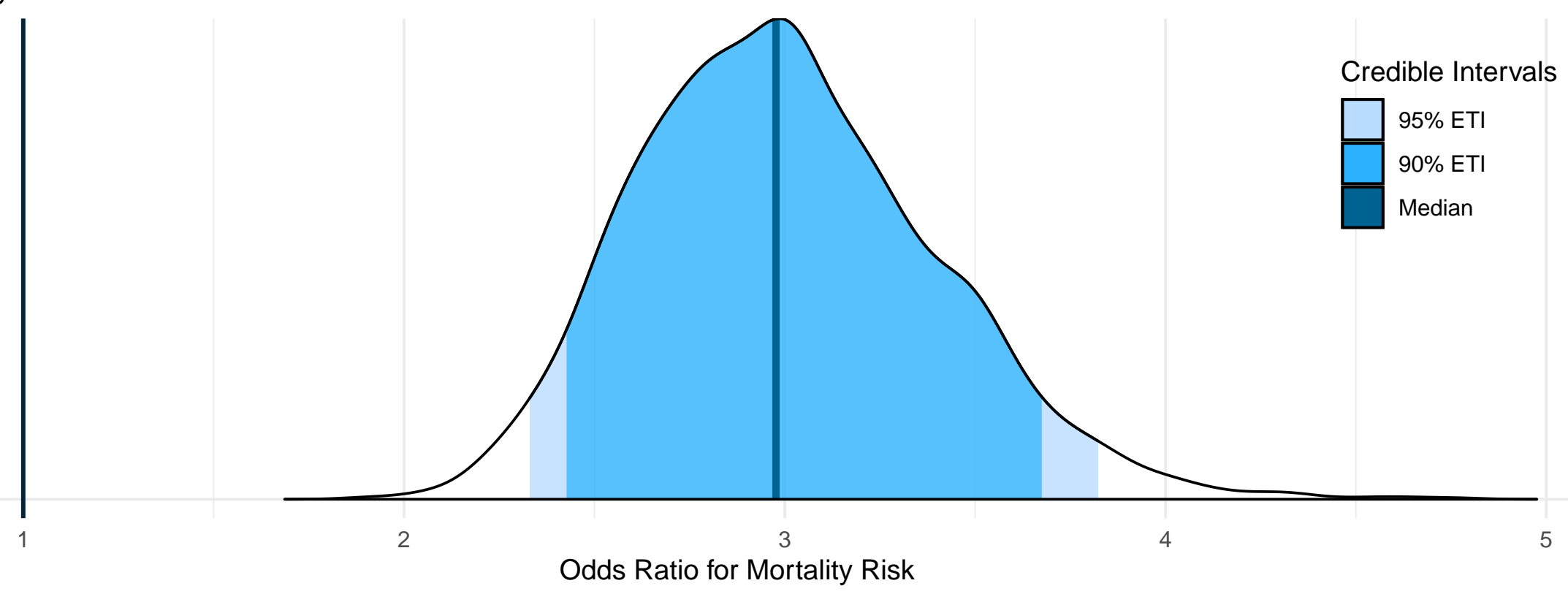

Credible Intervals

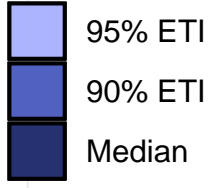

Median $95 \%$ ETI $90 \%$ ETI Median 